\title{
Grape seed proanthocyanidins inhibit colon cancer-induced angiogenesis through suppressing the expression of VEGF and Ang1
}

\author{
SHUANGSHENG HUANG $^{1 *}$, NINGGANG YANG ${ }^{2 *}$, YUANYUAN LIU ${ }^{3}$, JING GAO $^{1}$, TAO HUANG ${ }^{1}$, \\ LAMEI HU ${ }^{4}$, JIN ZHAO ${ }^{1}$, YONGQUAN LI ${ }^{1}$, CAILI LI ${ }^{1}$ and XIAOSU ZHANG ${ }^{1}$ \\ ${ }^{1}$ Medical College of Northwest University for Nationalities, Lanzhou; ${ }^{2}$ Department of Urology, \\ Lanzhou First People's Hospital, Lanzhou; ${ }^{3}$ Institute of Modern Physics, Chinese Academy of Sciences, Lanzhou; \\ ${ }^{4}$ Institute of Pharmacology, School of Basic Medical Science, Lanzhou University, Lanzhou, Gansu, P.R. China
}

Received July 20, 2012; Accepted September 4, 2012

DOI: $10.3892 /$ ijmm.2012.1147

\begin{abstract}
Tumor cells trigger angiogenesis through overexpression of various angiogenic factors including vascular endothelial growth factor (VEGF) and angiopoietin 1 (Ang1). Therefore, inhibition of the expression of both VEGF and Ang1, the initial step of tumor angiogenesis, is a promising strategy for cancer chemoprevention and therapy. Grape seed proanthocyanidins (GSPs) are widely consumed dietary supplements that have antitumor activity. Due to their polymeric structure, GSPs are poorly absorbed along the gastrointestinal tract and can reach the colon at high concentrations, allowing these chemicals to act as chemopreventive agents for colon cancer. In the present study, we found that GSPs inhibited colon tumor-induced angiogenesis and, thus, the growth of colon tumor xenografts on the chick chorioallantoic membranes. The mechanisms of their action were related to inhibiting the expression of both VEGF and Ang1 through scavenging reactive oxygen species. Previous studies have demonstrated that the chemopreventive effects of GSPs on colon cancer are associated with their growth inhibitory and apoptosis-inducing effects. Our results demonstrate another mechanism by which GSPs inhibit colon tumor growth, which will be helpful for developing GSPs as a pharmacologically safe angiopreventive agent against colorectal cancer.
\end{abstract}

\section{Introduction}

Angiogenesis is the formation of new blood vessels from preexisting ones, and is required for tumor growth and metastasis.

Correspondence to: Dr Shuangsheng Huang, Medical College of Northwest University for Nationalities, 1 Xibei Xincun, Lanzhou, Gansu 730030, P.R. China

E-mail: huangshsh02@qq.com

*Contributed equally

Key words: grape seed proanthocyanidin, colon cancer, angiogenesis, vascular endothelial growth factor, angiopoietin 1, reactive oxygen species
Without new blood vessel formation, the tumor cannot grow larger than 1-2 mm in diameter (1). To develop, tumors must make an 'angiogenic switch' by perturbing the local balance between pro- and anti-angiogenic factors in the immediate environment of endothelial cells through the overexpression of proangiogenic growth factors, such as vascular endothelial growth factor (VEGF), basic fibroblast growth factor (bFGF) and angiopoietin (Ang) (2,3). As a general mechanism, oxidative stress is a common hallmark of numerous tumors. Tumor growth produces large amounts of reactive oxygen species (ROS) (4). Previous studies have shown that ROS trigger 'angiogenic switch' responses by inducing VEGF expression and matrix metalloproteinase activity (5). Thus, discovery of non-toxic antiangiogenic agents from nature antioxidants targeting to inhibit the production of proangiogenic growth factors, the initial step of angiogenesis, is a promising strategy for cancer therapy and prevention.

Colon cancer is the third most commonly diagnosed cancer in men and women, with 103,170 new cases and 51,690 deaths estimated to occur in 2012 in the United States (6). The incidence of colon cancer in China is lower than that in the western countries, but it has increased in recent years and become a substantial cancer burden in China, particularly in the more developed areas (7). Epidemiological studies have shown that the regular consumption of fruits and vegetables is associated with a reduced risk of cancer (8). The beneficial effects may be partly attributable to polyphenolic compounds which have antioxidant and free radical scavenging properties (9).

Grapes are one of the most widely consumed fruits in the world and are rich in polyphenols, of which about $60-70 \%$ are found in grape seeds as dimers, trimers and other oligomers of flavan-3-ols and commonly known as proanthocyanidins. Grape seed proanthocyanidins (GSPs) are widely consumed as a dietary supplement and possess chemopreventive and/or chemotherapeutic effects in various cell culture and animal models $(10,11)$. Pharmacokinetic studies have shown that, in vivo, GSPs can hardly be absorbed or metabolized during upper gastrointestinal tract transit, allowing these chemicals to reach the colon at high concentrations (12-14). This metabolic characteristic suggests that GSPs have a natural colon-targeting 
feature and are more adaptive to acting as a chemopreventive and chemotherapeutic agent for colon cancer. Epidemiological studies suggested an inverse relationship between the dietary consumption of proanthocyanidins and the risk of colorectal cancer $(15,16)$. This is also supported by both in vitro and experimental animal studies (17-25). These studies have shown that GSPs inhibit growth and induce the apoptosis of some colon caner cell lines in vitro and in vivo. Dietary-feeding of grape seed extract also prevents azoxymethane-induced colonic aberrant crypt foci formation in Fischer 344 rats (26), suggesting that grape seed extract could inhibit the early steps of colon carcinogenesis.

In the present study, we report that GSPs inhibit tumorinduced angiogenesis, and, thus, colon tumor growth by inhibiting the expression of VEGF and Ang1 through scavenging ROS. Our results provide a novel explanation for GSPs as an angiopreventive agent against colon cancer.

\section{Materials and methods}

Materials. GSPs, consisting of at least $95 \%$ proanthocyanidins, $1.8 \%$ proanthocyanidin B2 and $60 \%$ oligomers were purchased from Jianfeng Co. (Tianjin, China). Leibovitz's L-15 medium, MCDB131 medium, epithelial growth factor (EGF), hydrocortisone, sulforhodamine B (SRB) and 2',7'-dichlorofluorescein diacetate (DCFH-DA) were purchased from Sigma (St. Louis, MO, USA). Fetal bovine serum (FBS) was purchased from Lanzhou National HyClone Bio-Engineering Co., Ltd. (Lanzhou, China). Millicell cell culture inserts were purchased from Millipore Corp. (Bedford, MA, USA). VEGF and Ang1 ELISA kits were ordered from R\&D Systems (Minneapolis, MN, USA). Rabbit polyclonal anti-VEGF and rabbit polyclonal anti-Ang1 were purchased from Boster Bio-engineering Limited Co., Ltd. (Wuhan, China). MaxVision TM HRP-Polymer antiMouse/Rabbit IHC kit and DAB kit were purchased from Maixin Biological Technology, Ltd. (Fuzhou, China).

Cell culture. Human colon cancer SW620 cells were obtained from the Cell Bank of the Type Culture Collection of the Chinese Academy of Sciences (Shanghai, China) and were cultured in Leibovitz's L-15 medium supplemented with $10 \%$ FBS. Human microvascular endothelial cells (HMEC-1) were cultured in MCDB131 medium supplemented with $1.18 \mathrm{mg} / \mathrm{ml}$ $\mathrm{NaHCO}_{3}, 20 \%$ inactivated FBS, $10 \mathrm{ng} / \mathrm{ml} \mathrm{EGF}$ and $1 \mu \mathrm{g} / \mathrm{ml}$ hydrocortisone. All cells were cultured in a highly humidified atmosphere of $5 \% \mathrm{CO}_{2}$ at $37^{\circ} \mathrm{C}$. Fertilized White Leghorn chicken eggs were obtained from the Lanzhou Institute of Biological Products (Lanzhou, China) and were incubated at $37^{\circ} \mathrm{C}$ in a humidified egg incubator.

Cell viability assay. The viability of SW620 cells was determined in 96-well plates by the SRB method with some modifications (27). Briefly, exponentially growing cells were seeded in 96-well plates with the final volume $100 \mu \mathrm{l} /$ well. After $24 \mathrm{~h}$ of incubation, cells were treated with various concentrations of GSPs for the indicated times. The cultures were then fixed at $4^{\circ} \mathrm{C}$ for $1 \mathrm{~h}$ with ice-cold $50 \%$ trichloroacetic acid to give a final concentration of $10 \%$. Fixed cells were rinsed 5 times with deionized water and stained for $10 \mathrm{~min}$ with $0.4 \%$ SRB dissolved in $0.1 \%$ acetic acid. The wells were then washed 5 times with $0.1 \%$ acetic acid and left to dry overnight. The absorbed SRB was dissolved in $150 \mu$ l unbuffered $1 \%$ Tris base (pH 10.5). The absorbance of extracted SRB at $515 \mathrm{~nm}$ was measured on a microplate reader.

Chick chorioallantoic membrane (CAM) tumor formation assay $(28,29)$. Fertilized White Leghorn chicken eggs were incubated under conditions of constant humidity at a temperature of $37^{\circ} \mathrm{C}$. On Day 10 of incubation, a small hole was punched over the air sac in order to detach the CAM from the eggshell by gently exhausting, and then a square window was opened on the broad side of the egg, exposing the CAM. After the CAM was exposed, $40 \mu 1$ serum-free culture medium containing $1 \times 10^{6}$ SW620 cells were deposited on the CAM. The window was sealed with tape and the eggs were returned to the incubator. When the solid tumor began to vascularize after implantation for two days, GSP or vehicle was deposited locally each day. Five days later, CAM was photographed in ovo under a stereomicroscope and tumors were resected and weighed.

Enzyme-linked immunosorbent assay(ELISA). For the measurement of VEGF and Ang1 secretion, confluent SW620 cells (90-100\%) were cultured in serum-free media for $24 \mathrm{~h}$ in the absence or presence of GSP. Cell-free culture supernatants were harvested and used for the determination of VEGF and Ang1 levels using a human VEGF or Ang1 ELISA kit according to the manufacturer's instructions. The concentration of the VEGF and Ang1 in the samples was then determined by comparing the optical density of the samples to the standard curve.

Preparation of tumor conditioned medium (30). Tumor conditioned medium was prepared from the SW620 cell culture as follows: SW620 cells were grown to subconfluency (approximately 90\%). After being washed twice with D-Hanks, cells were incubated in MCDB131 medium supplemented with or without $100 \mu \mathrm{g} / \mathrm{ml} \mathrm{GSP}$ for $24 \mathrm{~h}$. The supernatant was then harvested, centrifuged at $2000 \mathrm{xg}$ at $4^{\circ} \mathrm{C}$ for $10 \mathrm{~min}$, filtersterilized through $0.22-\mu \mathrm{m}$ pore size filters and stored at $-20^{\circ} \mathrm{C}$ prior to use.

Cell migration assay. Cell migration was performed in millicell cell culture inserts using a polycarbonate filter with a pore size of $8 \mu \mathrm{m}$. HMEC- 1 cells $\left(2 \times 10^{5}\right)$ suspended in $0.4 \mathrm{ml}$ serum-free MCDB131 culture medium were added to the upper compartment of cell culture inserts. The lower compartment contained $0.6 \mathrm{ml}$ conditioned medium. Following incubation for $24 \mathrm{~h}$ at $37^{\circ} \mathrm{C}$, the nonmigrated cells on the upper surface of the membrane were removed with a cotton swab. The migrated cells on the lower surface of the membrane were fixed with methanol and then stained with $0.1 \%$ crystal violet. Images from randomly selected microscopic fields were obtained under light microscopy. Each sample was repeated three times.

Intracellular ROS staining. Intracellular ROS levels in both GSP-treated and control cells were measured by DCFH-DA assay as previously described (31). Briefly, sub-confluent SW620 cells were treated with different concentrations of GSP for $24 \mathrm{~h}$. Following incubation, cells were washed once with D-Hanks and stained with DCFH-DA $(10 \mu \mathrm{M})$ for $30 \mathrm{~min}$. Subsequently, cells were washed twice with D-Hanks to remove 


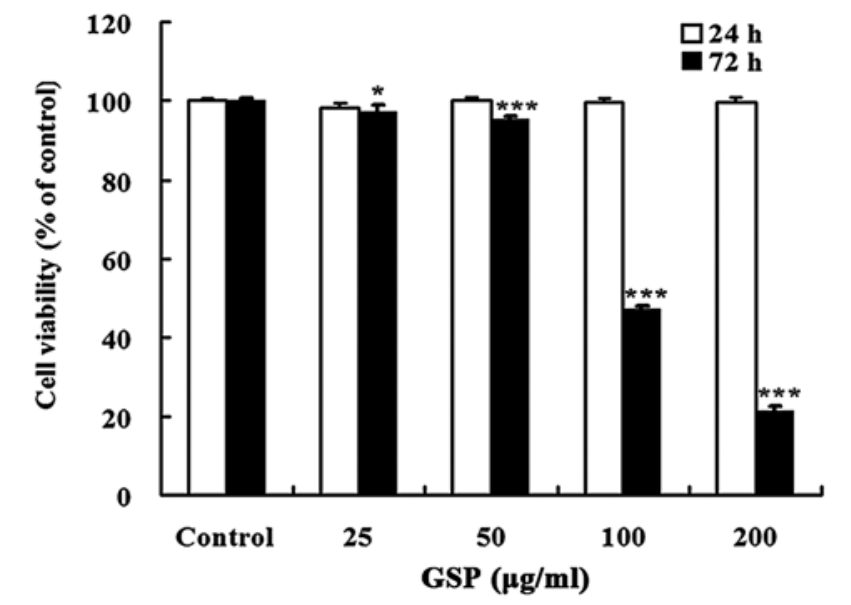

Figure 1. Effect of GSPs on the cell viability of SW620 cells. Following treatment with GSPs for the indicated times, cell viability was measured with the SRB assay. Results are representative of three independent experiments and are expressed as the means $\pm \mathrm{SD}$ of six cultures with the vehicle control as $100 \%$. ${ }^{*} \mathrm{P}<0.05,{ }^{* * *} \mathrm{P}<0.001$ vs. vehicle control.

the excess dye, and then $100 \mu \mathrm{l}$ of D-Hanks was added. The images were visualized and photographed under a fluorescent microscope. During the entire procedure with DCFH-DA, the plate was kept out of light to avoid fading of the fluoroprobe.

Immunohistochemical assay for VEGF and Angl expression. Following pretreatment with or without $100 \mu \mathrm{g} / \mathrm{ml} \mathrm{GSP}$ for $2 \mathrm{~h}$ and further incubation with or without $100 \mu \mathrm{M} \mathrm{H}_{2} \mathrm{O}_{2}$ for $24 \mathrm{~h}$, SW620 cells seeded on glass coverslips were fixed with $4 \%$ paraformaldehyde. The expression of both VEGF and Ang1 was detected with the immunohistochemical staining kit according to the manufacturer's instructions.

Statistical analysis. Results are expressed as the means \pm SD, and were analyzed using the Student's t-test. Values of $\mathrm{P}<0.05$ were considered to indicate statistically significant differences.

\section{Results}

Effect of GSPs on the cell viability of SW620 cells. GSP treatment for $72 \mathrm{~h}$ significantly inhibited cell viability in a dosedependent manner with an $\mathrm{IC}_{50}$ value of $116.44 \mu \mathrm{g} / \mathrm{ml}$. However, treatment with GSP $(25-200 \mu \mathrm{g} / \mathrm{ml})$ for $24 \mathrm{~h}$ did not change cell viability (Fig. 1). Therefore, all subsequent experiments were carried out with the treatment time not exceeding $24 \mathrm{~h}$.

GSPs inhibit tumor-induced angiogenesis and tumor growth in a xenografted chick chorioallantoic membrane (CAM) model. SW620 cells inoculated on chick CAM formed solid, avascular tumor within the first day. Two days after inoculation, the tumor became vascularized and grew rapidly. Five days later, numerous vessels developed radially around the tumor. Vessels at the tumor surface were clearly visible in the control group (Fig. 2A). Local treatment of the tumor from Day 3 to Day 7 (the day when SW620 cells were inoculated was designated as Day 1) with GSPs markedly inhibited

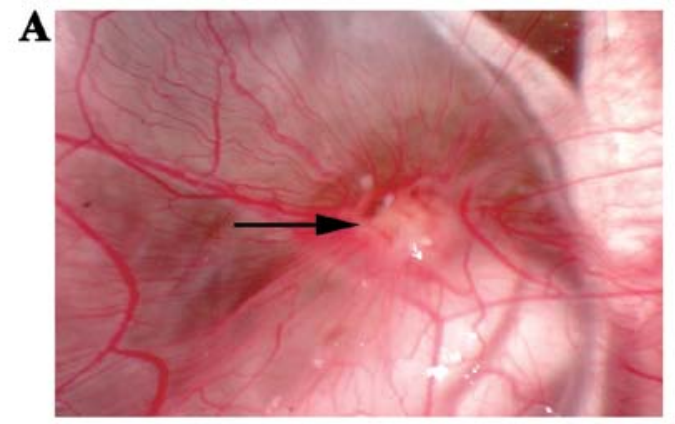

Control

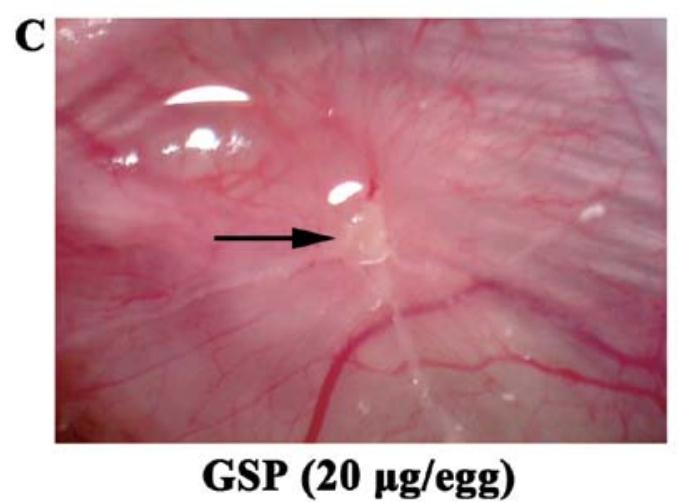

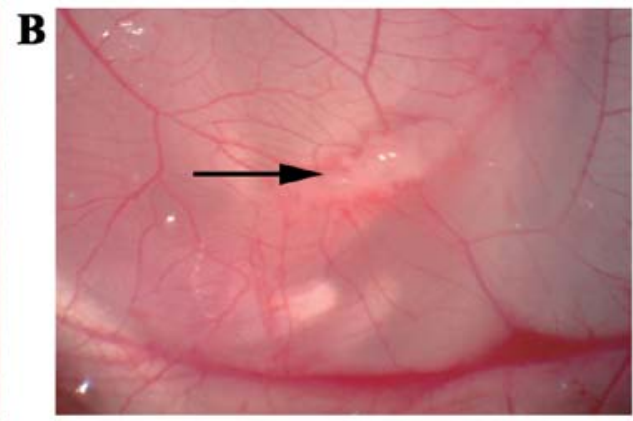

GSP (5 $\mu \mathrm{g} / \mathrm{egg})$

D

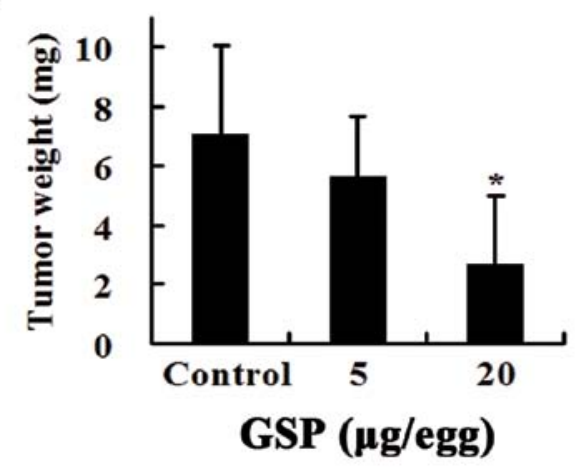

Figure 2. GSPs inhibit colon tumor angiogenesis and tumor growth on chick CAM. Cell suspensions of SW620 cells were delivered at $1 \times 10^{6}$ cells/embryo onto the top of CAM on Day 10. When the solid tumor became vascularized after a further 2 days of incubation, different concentrations of GSPs were placed on the CAMs every day for five days. Then patterns of angiogenesis toward tumor were photographed. (A) Vehicle control, (B) $5 \mu \mathrm{g} \mathrm{GSP} / \mathrm{egg}$, or (C) $20 \mu \mathrm{g} \mathrm{GSP} / \mathrm{egg}$. (D) Tumors were resected and weighed. The representative results from two independent experiments are shown and expressed as the means \pm SD ( $=6$ ). ${ }^{*} \mathrm{P}<0.05$ vs. vehicle control. 

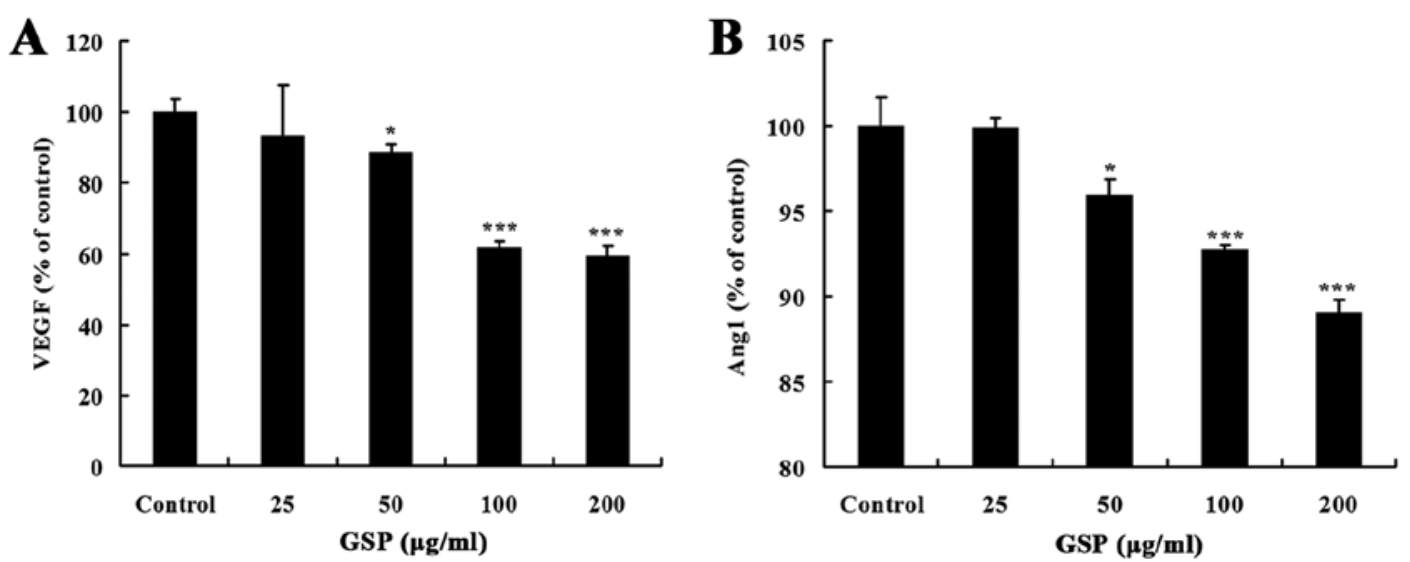

Figure 3. GSPs inhibit VEGF and Ang1 expression in SW620 cells. SW620 cells were seeded in 24-well plates and cultured to 90-100\% confluence. The old medium was discarded and fresh medium with or without GSPs was added. The cells were then incubated for $24 \mathrm{~h}$. (A) VEGF and (B) Ang1 in the supernatants of culture medium was determined with ELISA. The representative results from two independent experiments are shown and expressed as the means \pm SD $(\mathrm{n}=4) .{ }^{*} \mathrm{P}<0.05,{ }^{* * * *} \mathrm{P}<0.001$ vs. vehicle control.



Control

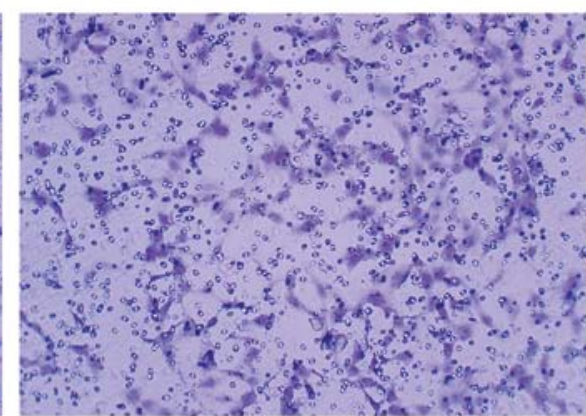

GSP $(100 \mu \mathrm{g} / \mathrm{ml})$

Figure 4. Conditioned medium collected from GSP-treated SW620 cells inhibits the migration of HMEC-1 cells. HMEC-1 cells were seeded in the upper chamber of millicells. The bottom chamber was filled with conditioned medium collected from (A) untreated or (B) $100 \mu \mathrm{g} / \mathrm{ml}$ GSP-treated SW620 cells. After approximately $24 \mathrm{~h}$, cells that passed through the membrane were fixed and stained. Representative photomicrographs are shown.

tumor-induced angiogenesis. The tumor appeared white (Fig. 2B and C). The growth of the tumor was also inhibited by GSP treatment (Fig. 2D). During the experiment, some chick embryos from both the control and the GSP-treated groups died before the end of the experiment and were not included in the results shown. However, there was no significant difference in the incidence of embryonic death between GSP-treated and control groups, indicating that the inhibitory effect of GSP on tumor-induced angiogenesis was not related to toxic effects.

GSPs inhibit expression of VEGF and Ang1 in SW620 cells. Solid tumors secrete various proangiogenic factors, such as VEGF and Ang1, to activate the nearest endothelial cells in the host tissue for neoangiogenesis (32). In the present study, we investigated whether GSPs could inhibit proangiogenic attributes of colon cancer SW620 cells. GSP treatment for $24 \mathrm{~h}$ inhibited both VEGF and Ang1 expression in a dose-dependent manner (Fig. 3). The inhibitory effect on VEGF expression was stronger than on Ang1 expression. These results suggest that the inhibitory effect of GSPs on tumor-induced angiogenesis is partly through suppressing the expression of angiogenic factors that initiate tumor angiogenesis in SW620 cells.
Conditioned medium from GSP-treated SW620 cells inhibits cell migration of HMEC-1 cells. Both VEGF and Ang1 are chemotactic factors specific for endothelial cells. To further verify that GSPs inhibit tumor-induced angiogenesis by suppressing the expression of both VEGF and Ang1 in tumor cells, we conducted endothelial cell migration assay to examine the effect of conditioned medium from either GSP-treated or untreated SW620 cells on endothelial cell migration. Conditioned medium collected from SW620 cells induced endothelial cell migration (Fig. 4). However, endothelial cell migration was suppressed by conditioned medium collected from $100 \mu \mathrm{g} / \mathrm{ml}$ GSP-treated SW620 cells. These results further verified that the inhibitory effect of GSP on tumorinduced angiogenesis is mediated by reducing the expression of proangiogenic factors in SW620 cells.

GSPs inhibit the expression of VEGF and Angl by reducing ROS production. Excessive ROS production has been confirmed to have a vital role in the induction of VEGF or Ang1 expression in many tumor cell lines. To investigate whether the antioxidant activity is part of the mechanisms by which GSPs suppress the expression of angiogenic factors in SW620 cells, we first used fluorescent probes DCFH-DA to 


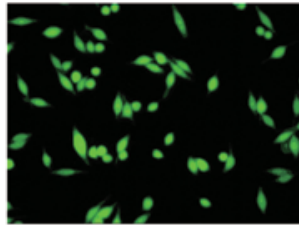

Control
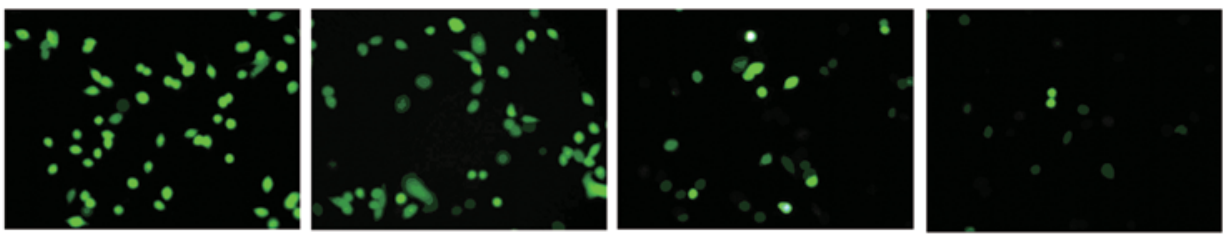

GSP $(25 \mu \mathrm{g} / \mathrm{ml})$ GSP $(50 \mu \mathrm{g} / \mathrm{ml})$ GSP $(100 \mu \mathrm{g} / \mathrm{ml})$ GSP $(200 \mu \mathrm{g} / \mathrm{ml})$

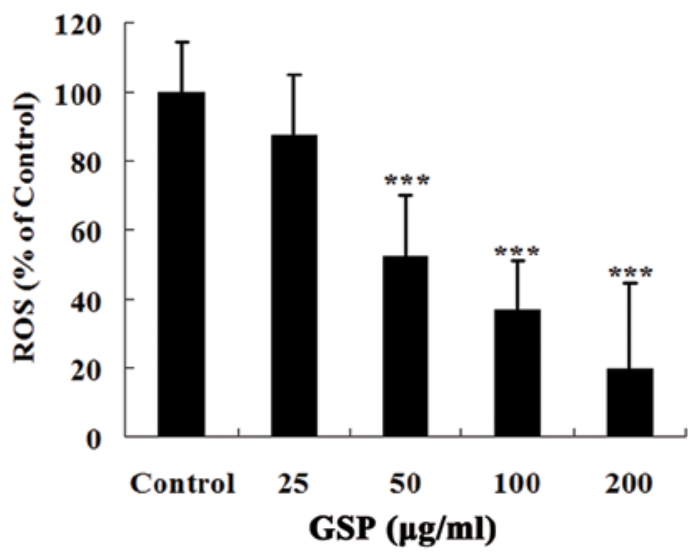

Figure 5. GSPs inhibit ROS production in SW620 cells. SW620 cells were treated with GSPs for 24 h and then incubated with DCFH-DA (10 $\mu$ M) for an additional $30 \mathrm{~min}$. The intracellular ROS level was captured under a fluorescence microscope, and the images were imported and analyzed using the ImageInside program. The representative results from three independent experiments are shown and expressed as the means $\pm \mathrm{SD}$. ${ }^{* * *} \mathrm{P}<0.001 \mathrm{vs}$. vehicle control.
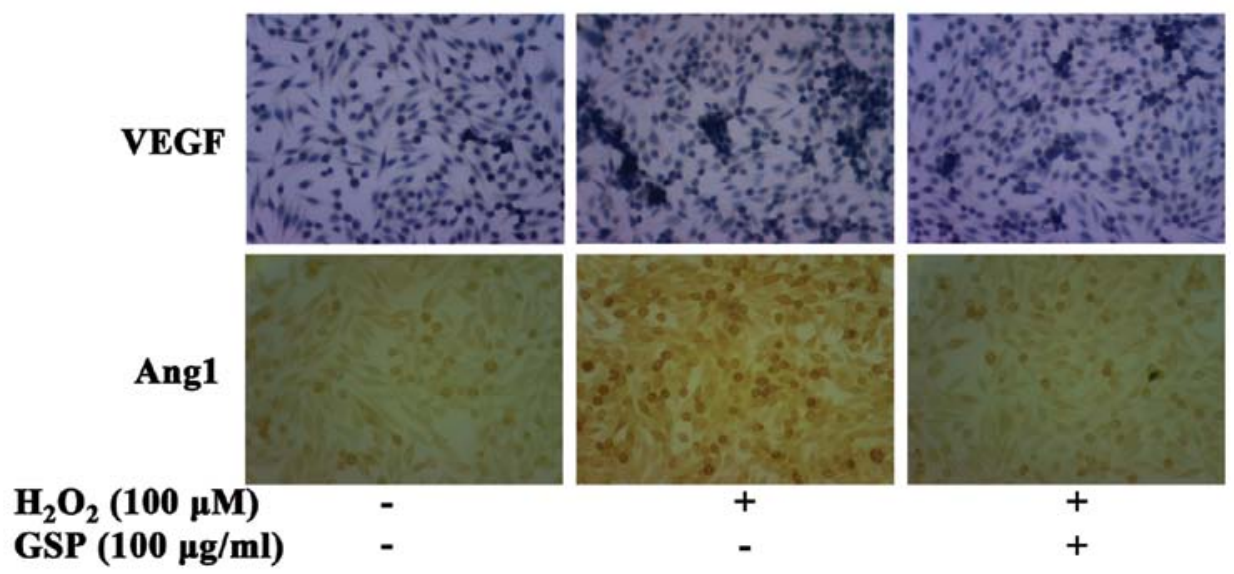
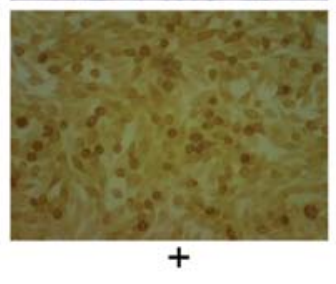

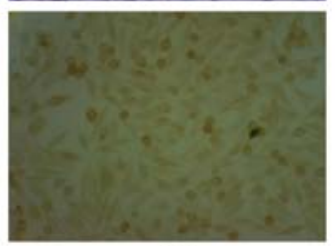

$+$

$+$

Figure 6. Inhibition of the expression of VEGF and Ang1 by GSPs depends on reducing the ROS production in SW620 cells. SW620 cells were treated with or without $100 \mu \mathrm{g} / \mathrm{ml} \mathrm{GSPs}$ for $2 \mathrm{~h}$ and then incubated with or without $100 \mu \mathrm{M} \mathrm{H}_{2} \mathrm{O}_{2}$ for $24 \mathrm{~h}$. The expression of VEGF and Ang1 were examined with immunohistochemical staining. Representative photomicrographs are shown.

detect the effect of GSPs on ROS production in SW620 cells. As shown in Fig. 5, colon cancer SW620 cells had a high level of ROS. Pretreatment with GSPs significantly inhibited ROS production in a concentration-dependent manner. Thus, our data suggest that GSPs can significantly reduce the high levels of intracellular produced ROS. To further verify that the antioxidant activity is part of the mechanisms by which GSPs suppress the expression of angiogenic factors, the effects of GSPs on $\mathrm{H}_{2} \mathrm{O}_{2}$-induced VEGF and Ang1 expression were examined with the immunohistochemical assay. As shown in Fig. 6, $100 \mu \mathrm{M}$ of $\mathrm{H}_{2} \mathrm{O}_{2}$ markedly induced both VEGF and Ang1 expression in SW620 cells. However, the expression levels of VEGF and Ang1 induced by $\mathrm{H}_{2} \mathrm{O}_{2}$ were suppressed by pretreatment with $100 \mu \mathrm{g} / \mathrm{ml}$ GSP.

\section{Discussion}

Tumor growth and metastasis are dependent on angiogenesis. Without new blood vessel formation, the tumor remains dormant and cannot grow larger than 2-3 $\mathrm{mm}$ in diameter. Tumors that grow beyond this size trigger angiogenesis by producing proangiogenic factors. Among these molecules, VEGF and Ang1 are the prime proangiogenic factors for sustaining tumor growth. Therefore, inhibition of both VEGF and Ang1 production, the initial step of tumor angiogenesis, is a promising strategy for cancer chemoprevention and therapy. In the current study, GSPs inhibited the colon tumor-induced angiogenesis and, thus, the growth of colon tumor xenograft on the chick CAM without any apparent sign of toxicity. 
Previous studies have demonstrated that the chemopreventive effects of GSPs for colon cancer are associated with their growth inhibitory and apoptosis-inducing effects. Our results, provide another mechanism by which GSPs inhibit colon tumor growth.

To better understand the mechanism by which GSPs inhibit the tumor-induced angiogenesis, we examined the effect of GSPs on the expression of proangiogenic factors by colon tumor cells. We found that GSP treatment exerted significant inhibitory effects on both VEGF and Ang1 expression. The result was further supported by the endothelial cell migration assay, which showed that conditioned medium from GSP-treate d SW620 cells exhibited greater inhibitory effects on endothelial cell migration than its untreated counterparts. This conclusion is consistent with previous findings that GSPs inhibit VEGF secretion from DU145 prostate cancer cells (33). Among many proangiogenic factors, both VEGF and Ang1 regulate different, but complementary, aspects of blood vessel growth in tumors by binding their receptor expressed on the endothelial cells. The former is responsible for new blood vessel formation, while the latter contributes to new blood vessel maturation and stabilization (32). Our observations indicate that GSPs inhibit both sprouting angiogenesis and maturation of blood vessels.

Both VEGF and Ang1 expression is regulated by several factors, including hypoxia-inducible factor-1 (HIF-1), in response to hypoxia (34). HIF-1 is a heterodimeric that consists of a constitutively expressed HIF-1 $\beta$ subunit and a HIF-1 $\alpha$ subunit, the expression of which is highly regulated. Under normal oxygen conditions, the HIF-1 $\alpha$ protein is hydroxylated by the prolyl hydroxylase enzymes (PHDs), thereby facilitating ubiquitination and subsequent proteasomal degradation (35). It has been reported that ROS generated from mitochondria are required for the stabilization of HIF-1 $\alpha$ (36). A previous study also showed that endogenous ROS regulate tumor-induced angiogenesis and tumor growth through HIF-1 $\alpha$ and VEGF expression in ovarian cancer cells (37). To understand whether the inhibition of GSPs on VEGF and Ang1 expression from SW620 cells may be mediated through their ROS scavenging activity, resulting blocking ROS/HIF-1 $\alpha /$ VEGF or Ang1 pathway, we examined the effect of GSPs on intracellular ROS levels at first. The results of our study showed that GSPs significantly reduced ROS levels in SW620 cells. Our results also showed that treatment with $100 \mu \mathrm{M}$ hydrogen peroxide stimulated both VEGF and Ang1 expression, while pretreatment with GSPs inhibited both VEGF and Ang1 expression. These results suggested that inhibition of VEGF and Ang1 expression by GSPs might partially attribute to their antioxidative activity.

It has been reported that GSPs, due to their polymeric structure, are poorly absorbed along the gastrointestinal tract and can reach the colon at concentrations of several hundred micromoles per liter, allowing these chemicals to act locally. These results, combined with our previous results that GSPs inhibit angiogenesis by inhibiting VEGF receptor 2 and receptor of Ang1 (Tie2) phosphorylation (38), indicate that GSPs are effective antiangiogenic agents by acting on both tumor and endothelial cells. Therefore, the results of the present study indicate that GSPs could be used as an effective, non-toxic antiangiogenic agent for colon cancer.

\section{Acknowledgements}

This study was supported by a grant from the National Natural Science Foundation of China (no. 30700142 to S.H.) and the Scientific Research Innovation Team of Northwest University for Nationalities (no. BMUCXTD-2011-1).

\section{References}

1. Folkman J: Role of angiogenesis in tumor growth and metastasis. Semin Oncol 29: 15-18, 2002.

2. Hanahan D and Folkman J: Patterns and emerging mechanisms of the angiogenic switch during tumorigenesis. Cell 86: 353-364, 1996.

3. Bergers $\mathrm{G}$ and Benjamin LE: Tumorigenesis and the angiogenic switch. Nat Rev Cancer 3: 401-410, 2003.

4. Szatrowski TP and Nathan CF: Production of large amounts of hydrogen peroxide by human tumor cells. Cancer Res 51: 794-798, 1991.

5. Arbiser JL, Petros J, Klafter R, et al: Reactive oxygen generated by Nox1 triggers the angiogenic switch. Proc Natl Acad Sci USA 99: 715-720, 2002.

6. Siegel R, Naishadham D and Jemal A: Cancer statistics, 2012. CA Cancer J Clin 62: 10-29, 2012.

7. Chen W, Zeng H, Zheng R, Zhang S and He J: Cancer incidence and mortality in China, 2007. Chin J Cancer Res 24: 1-8, 2012.

8. Block G, Patterson B and Subar A: Fruit, vegetables, and cancer prevention: a review of the epidemiological evidence. Nutr Cancer 18: 1-29, 1992.

9. Surh Y: Molecular mechanisms of chemopreventive effects of selected dietary and medicinal phenolic substances. Mutat Res 248: 305-327, 1999.

10. Nandakumar V, Singh T and Katiyar SK: Multi-targeted prevention and therapy of cancer by proanthocyanidins. Cancer Lett 269: 378-387, 2008.

11. Kaur M, Agarwal C and Agarwal R: Anticancer and cancer chemopreventive potential of grape seed extract and other grapebased products. J Nutr 139: 1806S-1812S, 2009.

12. Spencer JP, Chaudry F, Pannala AS, Srai SK, Debnam E and Rice-Evans C: Decomposition of cocoa procyanidins in the gastric milieu. Biochem Biophys Res Commun 272: 236-241, 2000.

13. Rios LY, Bennett RN, Lazarus SA, Rémésy C, Scalbert A and Williamson G: Cocoa procyanidins are stable during gastric transit in humans. Am J Clin Nutr 76: 1106-1110, 2002.

14. Fraga CG and Oteiza PI: Dietary flavonoids: Role of (-)-epicatechin and related procyanidins in cell signaling. Free Radic Biol Med 51: 813-823, 2011.

15. Prior RL and Gu L: Occurrence and biological significance of proanthocyanidins in the American diet. Phytochemistry 66: 2264-2280, 2005.

16. Rossi M, Negri E, Parpinel M, et al: Proanthocyanidins and the risk of colorectal cancer in Italy. Cancer Causes Control 21: 243-250, 2010.

17. Singletary KW and Meline B: Effect of grape seed proanthocyanidins on colon aberrant crypts and breast tumors in a rat dual-organ tumor model. Nutr Cancer 39: 252-258, 2001.

18. Nomoto $\mathrm{H}$, Iigo $\mathbf{M}$, Hamada $\mathrm{H}$, Kojima $\mathrm{S}$ and Tsuda $\mathrm{H}$ : Chemoprevention of colorectal cancer by grape seed proanthocyanidin is accompanied by a decrease in proliferation and increase in apoptosis. Nutr Cancer 49: 81-88, 2004.

19. Kaur M, Singh RP, Gu M, Agarwal R and Agarwal C: Grape seed extract inhibits in vitro and in vivo growth of human colorectal carcinoma cells. Clin Cancer Res 12: 6194-6202, 2006.

20. Engelbrecht AM, Mattheyse M, Ellis B, et al: Proanthocyanidin from grape seeds inactivates the PI3-kinase/PKB pathway and induces apoptosis in a colon cancer cell line. Cancer Lett 258: 144-153, 2007

21. Kaur M, Mandair R, Agarwal R and Agarwal C: Grape seed extract induces cell cycle arrest and apoptosis in human colon carcinoma cells. Nutr Cancer 60 (Suppl 1): S2-S11, 2008.

22. Hsu CP, Lin YH, Chou CC, et al: Mechanisms of grape seed procyanidin-induced apoptosis in colorectal carcinoma cells. Anticancer Res 29: 283-289, 2009.

23. Velmurugan B, Singh RP, Kaul N, Agarwal R and Agarwal C: Dietary feeding of grape seed extract prevents intestinal tumorigenesis in APC min/+ mice. Neoplasia 12: 95-102, 2010. 
24. Dinicola S, Cucina A, Pasqualato A, et al: Apoptosis-inducing factor and caspase- dependent apoptotic pathways triggered by different grape seed extracts on human colon cancer cell line Caco-2. Br J Nutr 104: 824-832, 2010.

25. Kaur M, Tyagi A, Singh RP, Sclafani RA, Agarwal R and Agarwal C: Grape seed extract upregulates p21 (Cip1) through redox-mediated activation of ERK1/2 and posttranscriptional regulation leading to cell cycle arrest in colon carcinoma HT29 cells. Mol Carcinog 50: 553-562, 2011.

26. Velmurugan B, Singh RP, Agarwal R and Agarwal C: Dietaryfeeding of grape seed extract prevents azoxymethane-induced colonic aberrant crypt foci formation in fischer 344 rats. Mol Carcinog 49: 641-652, 2010.

27. Vichai V and Kirtikara K: Sulforhodamine B colorimetric assay for cytotoxicity screening. Nat Protoc 1: 1112-1116, 2006.

28. Hagedorn M, Javerzat S, Gilges D, Meyre A, de Lafarge B, Eichmann A and Bikfalvi A: Accessing key steps of human tumor progression in vivo by using an avian embryo model. Proc Natl Acad Sci USA 102: 1643-1648, 2005.

29. Balke M, Neumann A, Kersting C, et al: Morphologic characterization of osteosarcoma growth on the chick chorioallantoic membrane. BMC Res Notes 3: 58, 2010.

30. Hewett PW: Identification of tumour-induced changes in endothelial cell surface protein expression: an in vitro model. Int $\mathrm{J}$ Biochem Cell Biol 33: 325-335, 2001.

31. Wang $\mathrm{H}$ and Joseph JA: Quantifying cellular oxidative stress by dichlorofluorescein assay using microplate reader. Free Radical Biol Med 27: 612-616, 1999.
32. Saharinen P, Eklund L, Pulkki K, Bono P and Alitalo K: VEGF and angiopoietin signaling in tumor angiogenesis and metastasis. Trends Mol Med 17: 347-362, 2011.

33. Singh RP, Tyagim AK, Dhanalakshmim S, Agarwalm R and Agarwalm C: Grape seed extract inhibits advanced human prostate tumor growth and angiogenesis and up-regulates insulinlike growth factor binding protein-3. Int J Cancer 108: 733-740, 2004.

34. Hickey MM and Simon MC: Regulation of angiogenesis by hypoxia and hypoxia-inducible factors. Curr Top Dev Biol 76: 217-257, 2006.

35. Kaelin WG Jr and Ratcliffe PJ: Oxygen sensing by metazoans: the central role of the HIF hydroxylase pathway. Mol Cell 30: 393-402, 2008

36. Bell EL, Klimova TA, Eisenbart J, et al: The Qo site of the mitochondrial complex III is required for the transduction of hypoxic signaling via reactive oxygen species production. J Cell Biol 177: 1029-1036, 2007.

37. Xia C, Meng Q, Liu LZ, Rojanasakul Y, Wang XR, Jiang BH: Reactive oxygen species regulate angiogenesis and tumor growth through vascular endothelial growth factor. Cancer Res 67: 10823-10830, 2007.

38. Huang SS, Yang NG, Liu YY, et al: Grape seed proanthocyanidins inhibit angiogenesis via the downregulation of both vascular endothelial growth factor and angiopoietin signaling. Nutrition Res 32: 530-536, 2012. 